\title{
Effective field theory calculations in open charm and char- monium production in media
}

\author{
Miguel Ángel Escobedo Espinosa ${ }^{1,2, *}$ \\ ${ }^{1}$ Department of Physics, P.O. Box 35, FI-40014 University of Jyväskylä, Finland \\ ${ }^{2}$ Instituto Galego de Física de Altas Enerxías (IGFAE), Universidade de Santiago de Compostela, \\ Galicia-Spain
}

\begin{abstract}
Heavy quarkonium related observables are very useful to obtain information about the medium created in relativistic heavy ion collisions. The interaction of charmonium or bottomonium with the hot QCD medium created in these collisions can be efficiently described with the use of non-relativistic Effective Field Theories. In this proceedings, I will review recent progress in understanding quarkonium dynamics in a thermal medium with the use of potential non-relativistic QCD (pNRQCD). First, I will discuss the medium modifications of the decay width and the binding energy. Using PNRQCD power counting it is possible to identify in which temperature regimes a potential model can describe these modifications and what is the dominant physical process behind the thermal corrections in each case. After that, I will discuss the combination of pNRQCD with open quantum systems techniques. This allows making predictions about the nuclear modification factor that can be compared with experimental measurements.
\end{abstract}

\section{Introduction}

The suppression of heavy quarkonium as a signal of the formation of a quark-gluon plasma was first proposed by Matsui and Satz in [1], it was based on the idea that the screening of the potential will suppress the interaction between the quark and the antiquark and therefore they will not bind. In perturbation theory screening means that we go from a Coulomb-type potential to a Yukawa-type

$$
V(r)=-\alpha_{s} \frac{e^{-m_{D} r}}{r},
$$

where $m_{D}$ is the Debye mass. As this mass gets larger the number of bound states that can be formed diminishes.

The phenomenon of screening tells us that some of the bound states that exist in a vacuum cannot exist in a medium above a given temperature, however, it can also happen that even if the state still exits in a medium its lifetime can be smaller than that of the fireball formed in heavy-ion collisions. The lifetime of the more bound quarkonium states in the vacuum is very long, however, this can change dramatically at finite temperature if new decay channels are open. The computation in [2] showed that the potential of quarkonium (defined as the Wilson loop in Minkowski space) has an imaginary part that is related to a medium induced

\footnotetext{
*e-mail: miguelangel.escobedo@usc.es
} 
decay width of quarkonium, this is the order of magnitude or bigger than what is found in the vacuum and quarkonium can be very efficiently dissociated.

There is a very important detail that we have neglected in the previous discussion. We have been talking about the potential of quarkonium in a medium but it is not obvious at all that the evolution of quarkonium fulfils a Schrödinger equation or that thermal modifications can be encoded in a modification of the potential. In fact, there is a long discussion on what is the correct input from Lattice QCD in order to extract quarkonium potential, the free energy or the internal energy (see [3] for a recent discussion). In order to give a systematic answer different groups started to apply non-relativistic Effective Field Theories (EFTs) to this problem $[4,5]$ because a similar strategy was already, and successfully, applied in the vacuum to answer similar problems.

Let us review now the non-relativistic EFTs used in the vacuum. There are three energy scales relevant to the study of quarkonium. The scale of the heavy quark mass $M$, called the hard scale. The scale of the typical three-momentum or inverse of the typical radius $\frac{1}{r}$, called the soft scale. Finally, the scale of the binding energy $E$ or ultrasoft scale.

- Non-relativistic QCD (NRQCD) [6, 7] is the theory that is obtained after integrating out the hard scale. Its degrees of freedom are (apart from gluons and light quarks) a field for heavy quarks and another for heavy antiquarks, which are normal spinors instead of bispinors. This EFT is often used to compute quarkonium production cross-sections.

- Potential NRQCD (pNRQCD) [8, 9] is the theory obtained from NRQCD when the soft scale is also integrated out. In this theory the degrees of freedom are a quantum field for colour singlets and another for colour octets, the reason is that gluons with ultrasoft energy can no longer distinguish the heavy quark and antiquark and they are only sensitive to the full-colour state of the combination. pNRQCD has been used to obtain predictions for the spectroscopy and the decays of quarkonium.

The question about the applicability region of a potential model gets a very precise answer in the EFT formalism. The evolution of the singlet at leading order is given by a Schrödinger equation and the potential is just a Wilson coefficient of pNRQCD that, as any parameter in a quantum field theory, is affected by renormalization. However, in pNRQCD there are also higher order contributions, suppressed by a factor $(r E)^{2}$, which can not be described by a potential model and that represent the interaction of the singlet field with ultrasoft gluons.

In order to apply a similar program at finite temperature, one has to recognize the energy scales induced by the medium, in a perturbative plasma we have the temperature $\mathrm{T}$ and the Debye mass $m_{D} \sim g T$. There is yet another smaller scale (of order $g^{2} T$ ) that is nonperturbative even in the limit $g \rightarrow 0$, but we are not taking it into account in the present discussion. Different physical situations will arise depending on the relation of these energy scales with the scales of heavy quarkonium physics.

\section{Binding energies and decay widths corrections from EFTs}

An object that contains much information about the physics of quarkonium in a medium is the time-ordered propagator of the singlet. It encodes the medium modifications of the dispersion relation (corrections to the mass and the decay width), and its knowledge allows to obtain the spectral function (which is useful to compare with Lattice QCD results). Note that this object is not directly related to the number of quarkonium states in a plasma, so it is not possible to compute $R_{A A}$ only using it. Here we summarize essential points about our current knowledge about this object (based on pNRQCD computations in $[4,5,10]$ ): 


\subsection{The case $\frac{1}{r} \gg T \gg E \gg m_{D}$}

This case was studied in detail in [11]. In this temperature regime neither the hard nor the soft scales are affected by the temperature, so we can use as a starting point the same pNRQCD Lagrangian as in the vacuum. This means that thermal effects are a perturbation. The first thermal corrections to the binding energy are of order $\alpha_{s} r^{2} T E^{2}$ and they include terms that can not be encoded in a potential which are similar to the Bethe logs that appear in the Lamb shift.

Regarding the decay width, it is dominated by a process called gluo-dissociation [12, 13], in this process the singlet field absorbs a gluon from the medium and turns into an octet. In this case, it also happens that part of the corrections cannot be encoded in a potential.

The results of [11] were compared with a lattice computation in [14] finding a reasonable agreement.

\subsection{The case $\frac{1}{r} \gg T \gg m_{D} \gg E$}

This case is conceptually quite similar to the previous one, the main differences are the following:

- Up to very small corrections compared to the leading order thermal effects, all medium modifications can be encoded in potential because all thermal scales are bigger than the binding energy.

- The decay width is dominated by inelastic parton scattering [15], this is the process in which a singlet scatters with a parton in the medium (that can be either a gluon or a quark) and as a result, an octet and a parton are obtained. This process is $\alpha_{s}$ suppressed with respect to gluo-dissociation, however at high temperatures the phase space for this process is much bigger. The EFT power counting correctly predicts that for $m_{D} \gg E$ this is the dominant process.

\subsection{The case $\frac{1}{r} \sim T$}

In this case, it is still true that the hard scale is unaffected by the medium so the $T=0$ NRQCD Lagrangian can still be used as a starting point. However, the matching between NRQCD and pNRQCD will be affected by the temperature. In consequence, the singlet potential will get thermal corrections. These corrections are suppressed by an additional power of $\alpha_{s}$ in perturbation theory but not by any ratio of energy scales as happens in the $\frac{1}{r} \gg T$ case, the physical reason is that now the particles in the medium can already resolve separately the quark from the antiquark.

The potential in this temperature regime (both real and imaginary part) was studied in the analogous case of muonic hydrogen [10] (that can be understood as an Abelian version of quarkonium). The cross-section and decay width in this regime for quarkonium were studied in [15].

\subsection{The case $T \gg \frac{1}{r} \sim m_{D}$}

In this case, both the matching between QCD and NRQCD and between NRQCD and pNRQCD are affected by the medium. From the point of view of particles with an energy of order $T$ the heavy quark and antiquark are seen as very separated entities. For this reason, the leading thermal modification to the NRQCD Lagrangian is just a mass redefinition that has a very little phenomenological impact [10]. However, the singlet potential gets a contribution 
from the scale $m_{D}$ that is of the same order as the vacuum part even in perturbation theory. The potential computed in EFT at this temperature regime $[4,5]$ coincides with the complex potential that was previously computed in [2].

\section{Open quantum systems combined with effective field theories. The $\frac{1}{r} \gg T$ case.}

The final goal is to understand quarkonium suppression in heavy-ion collisions, in other words, how the medium modifies the yield of bound states in nucleus-nucleus compared to $\mathrm{pp}$ collisions. In the pNRQCD framework, this is related to the quantity $\rho_{s}(x, y)=$ $\operatorname{Tr}\left(\rho S^{\dagger}(x) S(y)\right)$ which is the projection of the density matrix to the subspace with one singlet. For example, the probability to find a $\Upsilon(1 S)$ state is the projection of $\rho_{s}$ to the $\Upsilon(1 S)$ wave-function. The evolution of $\rho_{s}$ with time, under the assumption $\frac{1}{r} \gg T$, is described in $[16,17]$. The evolution of $\rho_{s}$ couples with that of the similar object $\rho_{o}$ (where o is for octet). In the limit in which all thermal scales $\left(T\right.$ and $\left.m_{D}\right)$ are bigger than the binding energy these coupled equations can be combined in a single Lindblad or GKSL equation $[18,19]$

$$
\partial_{t} \rho=-i[H(\gamma), \rho]+\sum_{k}\left(C_{k}(\kappa) \rho C_{k}^{\dagger}(\kappa)-\frac{1}{2}\left\{C_{k}^{\dagger}(\kappa) C_{k}(\kappa), \rho\right\}\right)
$$

The operators $C_{k}$ are called collapse operators and in this specific case they have the form

$$
C_{i}^{s o}(\kappa)=\sqrt{\frac{\kappa}{N_{c}^{2}-1}} r_{i}\left(\begin{array}{cc}
0 & 1 \\
\sqrt{N_{c}^{2}-1} & 0
\end{array}\right),
$$

for the transition between singlets and octets, and

$$
C_{i}^{o o}(\kappa)=\sqrt{\frac{\left(N_{c}^{2}-4\right) \kappa}{2\left(N_{c}^{2}-1\right)}} r_{i}\left(\begin{array}{ll}
0 & 0 \\
0 & 1
\end{array}\right),
$$

for the transition between different octets states. This structure has a simple physical interpretation as a singlet that absorbs or emits a gluon will always turn into an octet while an octet doing the same can either turn into a singlet or remain being an octet. The Hamiltonian that enters the Lindblad equation can be written as

$$
H(\gamma)=\left(\begin{array}{cc}
h_{s} & 0 \\
0 & h_{o}
\end{array}\right)+\frac{r^{2}}{2} \gamma\left(\begin{array}{cc}
1 & 0 \\
0 & \frac{N_{c}^{2}-2}{2\left(N_{c}^{2}-1\right)}
\end{array}\right),
$$

where $h_{s}$ and $h_{o}$ are the corresponding $\mathrm{T}=0$ Hamiltonians.

In eq. (2), two non-perturbative parameters, that are in principle calculable using Lattice QCD, encode all the needed information about the medium. They are

$$
\kappa=\frac{g^{2}}{6 N_{c}} \operatorname{Re} \int_{-\infty}^{+\infty} d s\left\langle\mathrm{~T} E^{a, i}(s, \mathbf{0}) E^{a, i}(0, \mathbf{0})\right\rangle
$$

and

$$
\gamma=\frac{g^{2}}{6 N_{c}} \operatorname{Im} \int_{-\infty}^{+\infty} d s\left\langle\mathrm{~T} E^{a, i}(s, \mathbf{0}) E^{a, i}(0, \mathbf{0})\right\rangle .
$$

To our knowledge, no Lattice computation on $\gamma$ is available. The results of the perturbative computation of the self-energy in [5] imply that $\gamma=-2 \zeta(3) C_{F}\left(\frac{4}{3} N_{c}+n_{f}\right) \alpha_{s}^{2}\left(\mu_{T}\right) T^{3} \approx$ 

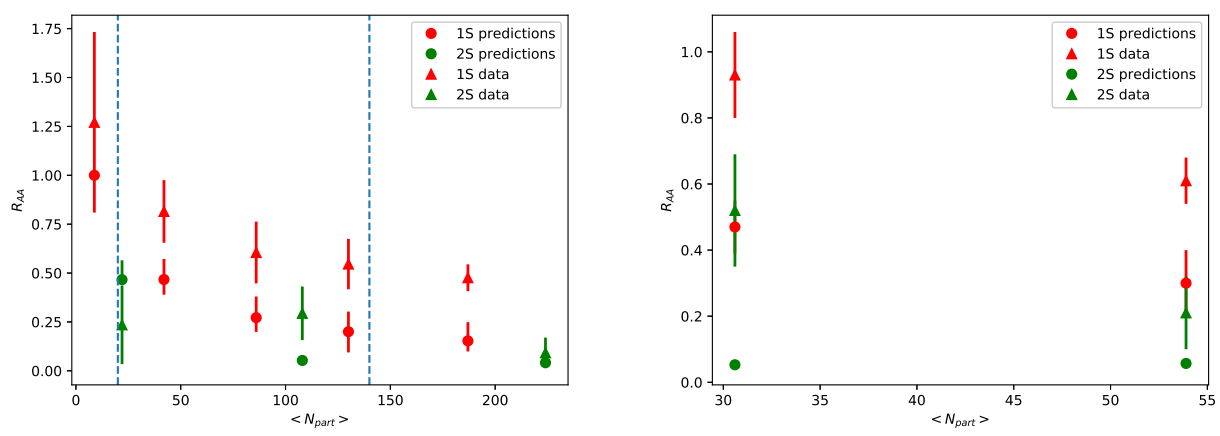

Figure 1. Suppression factor of $\Upsilon(1 S, 2 S)$ as a function of centrality (given by $\left.\left\langle N_{\text {part }}\right\rangle\right)$ in $\mathrm{PbPb}$ collisions. Left: comparison of our predictions with the experimental results of [23] at $\sqrt{s_{N N}}=2.76 \mathrm{TeV}$. The vertical dashed lines highlight the window in which we expect our approximations to be valid. Right: the comparison of our prediction with the experimental results of [24] at $\sqrt{s_{N N}}=5.02 \mathrm{TeV}$.

$-6.3 T^{3} . \kappa$ is the heavy quark diffusion constant that has a very important role also in the physics of open heavy flavor. There are many Lattice QCD computations of this quantity in the literature. To our knowledge, the most recent one is [20]. In a future publication, we will try to obtain $\kappa$ and $\gamma$ from lattice results on the mass shift and decay width of quarkonium [21].

In order to make some predictions we also need to give some initial conditions and some information about the thermal evolution of the medium. We assume that the system thermalizes suddenly at $t_{0}=0.6 \mathrm{fm}$ and afterwards it follows a Bjorken-type evolution [22]. Regarding the initial conditions, we assume that at $t=0$ both $\rho_{s}$ and $\rho_{o}$ are proportional to a Dirac delta in $r$, because the production process happens at very small distances, and that $\frac{\rho_{o}}{\rho_{s}}=\alpha_{s}$. We chose these naive assumptions in order to reduce the computational cost. However, we plan to improve these aspects in the future. In fig. 1 we show the results of applying Eq. (2) together with the value of $\kappa$ of [20] and assuming $\gamma=0$ and we compare them with experimental results.

\section{Outlook and conclusions}

We have presented an overview of the application of non-relativistic EFTs to the study of quarkonium suppression. In section 3 we have discussed how this framework can be applied to the computation of $R_{A A}$. We believe this is a very promising approach. However, to produce more accurate predictions of $R_{A A}$, improvements are needed in certain aspects:

- We need to extend the formalism to other temperature regimes (like for example $T \gg 1 / r$, we did study the case $1 / r \gg T \gg E \gg m_{D}$ that was not discussed here ). This will allow to study a wider range of bound states, collision energies and centralities.

- We need to reduce the computational cost. This will allow to use state-of-the-art hydrodynamics simulation (event-by-event initial conditions...).

- It is important also to model more accurately the initial distribution of quarkonium.

In conclusion, we have quite good understanding of the physics in thermal equilibrium. We can compute thermal corrections in perturbation theory, in some cases up to next-to-leading 
order, of the binding energy and the decay width. We have reported recent advances in understanding the evolution of quarkonium in an out-of-equilibrium medium and how this can be used to make prediction of the nuclear modification factor observed in experiments.

\section{Acknowledgements}

I want to acknowledge the collaboration of Nora Brambilla, Jacoppo Ghiglieri, Joan Soto and Antonio Vairo in several of the works discussed here. I have been supported by the Academy of Finland project 297058 and 303756, by Ministerio de Ciencia e Innovacion of Spain under project FPA2017-83814-P and Maria de Maetzu Unit of Excellence MDM-2016- 0692; by Xunta de Galicia and FEDER.

\section{References}

[1] T. Matsui, H. Satz, Phys. Lett. B178, 416 (1986)

[2] M. Laine, O. Philipsen, P. Romatschke, M. Tassler, JHEP 03, 054 (2007), hep-ph/0611300

[3] H. Satz, Eur. Phys. J. C75, 193 (2015), 1501.03940

[4] M.A. Escobedo, J. Soto, Phys. Rev. A78, 032520 (2008), 0804.0691

[5] N. Brambilla, J. Ghiglieri, A. Vairo, P. Petreczky, Phys. Rev. D78, 014017 (2008), 0804.0993

[6] W.E. Caswell, G.P. Lepage, Phys. Lett. 167B, 437 (1986)

[7] G.T. Bodwin, E. Braaten, G.P. Lepage, Phys. Rev. D51, 1125 (1995), [Erratum: Phys. Rev.D55,5853(1997)], hep-ph/9407339

[8] A. Pineda, J. Soto, Nucl. Phys. Proc. Suppl. 64, 428 (1998), [,428(1997)], hep-ph/9707481

[9] N. Brambilla, A. Pineda, J. Soto, A. Vairo, Nucl. Phys. B566, 275 (2000), hep-ph/9907240

[10] M.A. Escobedo, J. Soto, Phys. Rev. A82, 042506 (2010), 1008. 0254

[11] N. Brambilla, M.A. Escobedo, J. Ghiglieri, J. Soto, A. Vairo, JHEP 09, 038 (2010), 1007.4156

[12] N. Brambilla, M.A. Escobedo, J. Ghiglieri, A. Vairo, JHEP 12, 116 (2011), 1109.5826

[13] D. Kharzeev, H. Satz, Phys. Lett. B334, 155 (1994), hep-ph/9405414

[14] G. Aarts, C. Allton, S. Kim, M.P. Lombardo, M.B. Oktay, S.M. Ryan, D.K. Sinclair, J.I. Skullerud, JHEP 11, 103 (2011), 1109. 4496

[15] N. Brambilla, M.A. Escobedo, J. Ghiglieri, A. Vairo, JHEP 05, 130 (2013), 1303.6097

[16] N. Brambilla, M.A. Escobedo, J. Soto, A. Vairo, Phys. Rev. D96, 034021 (2017), 1612.07248

[17] N. Brambilla, M.A. Escobedo, J. Soto, A. Vairo, Phys. Rev. D97, 074009 (2018), 1711.04515

[18] G. Lindblad, Commun. Math. Phys. 48, 119 (1976)

[19] V. Gorini, A. Kossakowski, E.C.G. Sudarshan, J. Math. Phys. 17, 821 (1976)

[20] A. Francis, O. Kaczmarek, M. Laine, T. Neuhaus, H. Ohno, Phys. Rev. D92, 116003 (2015), 1508.04543

[21] N. Brambilla, M.A. Escobedo, A. Vairo, P. Vander Griend (2019), TUM-EFT 122/18

[22] J.D. Bjorken, Phys. Rev. D27, 140 (1983)

[23] V. Khachatryan et al. (CMS), Phys. Lett. B770, 357 (2017), 1611.01510

[24] A.M. Sirunyan et al. (CMS) (2018), 1805.09215 